\title{
Influence of a Chern-Simons term in the dynamical fermion masses in reduced or pseudo QED
}

\author{
Juan Angel Casimiro Olivares $\odot,{ }^{1}$ Luis Albino, ${ }^{2}$ Ana Julia Mizher, ${ }^{2,3}$ and Alfredo Raya $\circledast^{1,3}$ \\ ${ }^{1}$ Instituto de Física y Matemáticas, Universidad Michoacana de San Nicolás de Hidalgo. \\ Edificio C-3, Ciudad Universitaria. Francisco J. Míjica s/n Col. Felícitas del Río. \\ C. P. 58040, Morelia, Michoacán, Mexico \\ ${ }^{2}$ Instituto de Física Teórica, Universidade Estadual Paulista, \\ Rua Dr. Bento Teobaldo Ferraz, 271 - Bloco II, 01140-070 São Paulo, SP, Brazil \\ ${ }^{3}$ Centro de Ciencias Exactas-Universidad del Bio-Bio. \\ Avda. Andrés Bello 720, Casilla 447, Chillán, Chile
}

(Received 24 September 2020; accepted 4 November 2020; published 24 November 2020)

\begin{abstract}
Mixed-dimensional theories have been used to describe condensed matter systems where fermions are constrained to a plane while the gauge fields they interact with remain four dimensional. Here we investigate dynamical breaking of chiral symmetry in the framework of a mixed-dimensional theory that has been successful in describing graphene and other planar Dirac materials and has been dubbed pseudo or reduced quantum electrodynamics. We explore the interplay between the gauge and fermion sectors when a Chern-Simons term is considered and, by exploring the tensor structure of the fermion self-energy, we show that the radiative corrections may induce both a Dirac and a Haldane mass term. Furthermore, by solving the corresponding Schwinger-Dyson equation in a suitable truncation, we nonperturbatively explore the dynamical generation of fermion masses for different values of the electromagnetic coupling and the Chern-Simons coefficient. We also show that for definite parameter values, the contributions from each chirality cancel out and the chiral symmetry may be restored. Possible implications of this result for physical systems, in particular on what concerns the chiral magnetic effect, are discussed.
\end{abstract}

DOI: 10.1103/PhysRevD.102.096023

\section{INTRODUCTION}

Quantum electrodynamics in two spatial and one temporal dimension $\left(\mathrm{QED}_{3}\right)$ has been intensively studied in the past mainly because it shares two important features with quantum chromodynamics (QCD): chiral symmetry breaking (CSB) and confinement $[1,2]$. This allows to interpret such a theory as a QCD toy model and has been widely explored in the literature [3-26]. Under laboratory conditions of temperature and pressure the chiral symmetry of QCD is spontaneously broken. This symmetry breaking dynamically generates the masses of the constituent quarks and is ultimately responsible for the masses of light baryons and pseudoscalar mesons. However, under extreme conditions of the external parameters this symmetry may be restored, making the constituent-quark masses vanish. Understanding the nature and dynamics of this phase transition is one of the major goals of heavy-ion physics,

Published by the American Physical Society under the terms of the Creative Commons Attribution 4.0 International license. Further distribution of this work must maintain attribution to the author(s) and the published article's title, journal citation, and DOI. Funded by SCOAP ${ }^{3}$. with deep implications on what we know about the early Universe.

In this context, $\mathrm{QED}_{3}$ has been used as a tool to explore CSB. Schwinger-Dyson equations (SDEs) offer a suitable nonperturbative framework to capture the essence of this symmetry breaking, considering improved dressing of vertices and propagators. In a $1 / N$ expansion for pure $\mathrm{QED}_{3}$, it has been shown that in most cases there is a critical number of fermion flavors, above which the chiral symmetry breaking is restored [3,5,6,27]. Beyond this approximation, refined vertices and vacuum polarization effects agree with these findings in general, refining the details of the critical line [17]. These results reveal that what actually defines the existence of this criticality is the infrared behavior of the fermion wave function renormalization [20].

Physics on a plane is interesting on its own. It permits the inclusion of a Chern-Simons (CS) term in the gauge sector which induces fractional (anyon) statistic and parity (and time-reversal) noninvariance, among other interesting effects (see, for instance, Refs. [28-30] and references therein). Regarding CSB in $\mathrm{QED}_{3}$, the impact of adding the CS term in the photon sector was done by using different truncations of the SDE either in an appropriate nonlocal 
gauge [31-33] or in the Landau gauge [34]. These results show that, besides a critical number of families, there is also a critical value for the Chern-Simons parameter $\theta$ which induces chiral symmetry restoration. A critical line as a function of both parameters can be defined, and the literature [31-34] points towards a first-order transition.

With the experimental realization of graphene in 2004, $\mathrm{QED}_{3}$ met a more direct application. Besides being constrained to a plane, the charge carriers in this material in the low-energy regime obey a linear dispersion relation and carry a chirality degree of freedom. Because of this, $\mathrm{QED}_{3}$ was identified as the continuous limit of the tight-bind description of monolayer graphene [35]. This relativisticlike character of the quasiparticles in graphene-which subsequently was found in a few other Dirac/Weyl materials (see, for instance, Ref. [36] and references therein) — has opened the possibility of testing in a tabletop environment certain phenomena that were initially predicted in the context of high-energy physics, like the Klein paradox [37] and the Zitterbewegung [38].

Although $\mathrm{QED}_{3}$ has been shown to correctly describe the charge carriers in Dirac materials, interactions between these electrons and external electromagnetic fields cannot be correctly described by the full-fledged dimensionally reduced theory including gauge fields in $(2+1)$ dimensions. This is due to the fact that, although the fermionic quasiparticles are constrained to the plane, the gauge fields live in a bulk of higher dimensions and it is necessary to consider a mixed-dimensional theory to correctly describe this interaction. A procedure to deal with this condition was developed considering a four-dimensional gauge field interacting with fermion fields living in the boundary sheet $x_{3}=0$. This method was first appeared in Ref. [39] as pseudo-QED (PQED), and was later developed in Ref. [40], being dubbed reduced QED (RQED). We adopt the notation $\mathrm{P}(\mathrm{R}) \mathrm{QED}$.

Here, we investigate chiral symmetry breaking in the framework of $\mathrm{P}(\mathrm{R}) \mathrm{QED}$. This was done previously in the literature [40-42], but in the present work we focus on the influence of the Chern-Simons term on the dynamical mass generation in $\mathrm{P}(\mathrm{R}) \mathrm{QED}$ and its consequences for chiral symmetry. A pioneering investigation of CSB critical coupling in $\mathrm{QED}_{4}$ was done in Ref. [43], where the author found that CSB occurs above a critical coupling $\alpha_{c}=\pi / 3$. In Refs. $[44,45]$ the authors proposed that the dynamically generated mass should obey a scaling law $\propto \Lambda e^{-\pi / \sqrt{\alpha / \alpha_{c}-1}}$, what is known as Miransky scaling. In Refs. $[41,42,46]$ the authors performed a similar calculation for pure $\mathrm{P}(\mathrm{R}) \mathrm{QED}$, obtaining a critical value $\alpha_{c}=\pi / 8$. Thermal effects were further considered in Refs. [41,46] and it was explicitly shown how $\alpha_{c}$ depends on the temperature.

Our goal in the present paper is to explore the parameter space and detect for which values of the coupling and the CS- $\theta$ parameter CSB occurs. Perturbative [47] and nonperturbative features of the problem [48] have already been considered. For our purposes, we have organized the remainder of the manuscript as follows. In Sec. II we present the Lagrangian and discuss the Feynman rules. In Sec. III we solve the corresponding Schwinger-Dyson equation under the rainbow-ladder approximation in Landau gauge, neglecting wave function renormalization effects. We observe that, for suitable values of the parameters, two types of mass terms emerge. Finally, in Sec. IV we draw our conclusions and comment on possible implications of our results on high-energy-related condensed matter phenomena.

\section{LAGRANGIAN AND FEYNMAN RULES}

$\mathrm{P}(\mathrm{R}) \mathrm{QED}$ can be defined through the action

$$
\mathcal{S}_{\mathrm{QED}_{4}}=\int \mathrm{d}^{4} x\left[-\frac{1}{4} F_{\mu \nu} F^{\mu \nu}+e j_{\mu} A^{\mu}\right]+S_{\mathrm{gf}},
$$

which describes a four-dimensional gauge field interacting with a three-dimensional fermionic conserved current, given by

$$
j^{\mu}= \begin{cases}i \bar{\psi} \gamma \gamma^{\mu} \psi \delta\left(x_{3}\right) & \text { for } \mu=0,1,2, \\ 0 & \text { for } \mu=3 .\end{cases}
$$

Fermion dynamics can be included by adding up a threedimensional kinetic term $\int \mathrm{d}^{3} x \bar{\psi} i \not \partial \psi$.

The procedure consists in integrating out the fourdimensional gauge field, followed by an integration over the third space direction, perpendicular to the sheet where the fermion dynamics evolves. After that, one reintroduces the gauge fields, where the new field is consistently defined in three dimensions. This procedure leads to the following effective action:

$\mathcal{S}_{\mathrm{RQED}_{3}}=\int \mathrm{d}^{3} x\left[\frac{1}{2} F_{\mu \nu} \frac{1}{\sqrt{-\square^{2}}} F_{\mu \nu}+\bar{\psi} i \not D \psi\right]+S_{\mathrm{gf}}$,

where $F_{\mu \nu}$ is the electromagnetic field tensor, $A^{\mu}$ contained in the covariant derivative is the (three-dimensional) gauge field, and $\psi$ is the (four-component) fermion field. $S_{\mathrm{gf}}$ is a gauge-fixing term. Although $\mathrm{P}(\mathrm{R}) \mathrm{QED}$ is notably nonlocal, it has been shown that it respects causality and its Green functions are well defined [49].

Working with a linear gauge fixing, we can deduce propagators and vertices from the following Lagrangian:

$$
\mathcal{L}=-\frac{1}{4} F^{\mu \nu} \frac{2}{(-\square)^{1 / 2}} F_{\mu \nu}+\bar{\psi}\left(i \gamma^{\mu} \partial_{\mu}+e \gamma^{\mu} A_{\mu}\right) \psi+\frac{1}{2 \zeta}(\partial \cdot A)^{2},
$$

where indices run from 0 to $2, e$ is the electric charge, $(-\square)$ is the d'Alembertian operator, which appears in the Lagrangian with fractional power, and the last term is 
the gauge-fixing term with $\zeta$ being a gauge-fixing parameter.

The Euclidean Clifford algebra associated with the gamma matrices, $\left\{\gamma_{\mu}, \gamma_{\nu}\right\}=2 \delta_{\mu \nu}$, has two possible irreducible two-dimensional representations. A parity transformation swaps massive fermions from one representation into the other so that none of them are parity invariant. Due to the fact that in odd dimensions it is not possible to define a $\gamma_{5}$ matrix-one that is constructed from the anticommuting matrices in a given representation and anticommutes with all of them-it is not possible to define chirality. It is possible, however, to merge the particles in different representations and define a new, reducible, fourcomponent representation [50]. This is actually the usual procedure to define chirality in graphene [35], where the degrees of freedom of sublattices and valleys are represented in a single four-component structure and chirality can then be defined as associated to the latter.

In what follows we consider the chiral limit, which amounts to setting all fermion masses to zero. Furthermore, when solving the Schwinger-Dyson equation for the fermion propagator, we find it convenient to work with the four-component reducible representation of the fermion fields, as explained below.

The photon propagator derived from Eq. (4) is

$$
\Delta_{\mu \nu}^{0}(q)=\frac{1}{2 q}\left(\delta_{\mu \nu}-\frac{q_{\mu} q_{\nu}}{q^{2}}\right)+\frac{\zeta}{q^{2}} \frac{q_{\mu} q_{\nu}}{q^{2}} .
$$

One can notice that it presents a softer infrared behavior than the photon propagator in QED in four dimensions. The massless fermion propagator is given by

$$
S_{0}^{-1}(p)=-\gamma^{\mu} p_{\mu} .
$$

The focus of this work is to explore the consequences of coupling $\mathrm{P}(\mathrm{R}) \mathrm{QED}$ to a Chern-Simons term. CS belongs to a class of gauge theories that can only be defined in odd dimensions. Besides being a breakthrough in mathematical physics, they have been useful to describe several condensed matter phenomena, from the fractional quantum Hall effect (see Refs. [28,29] and references within) to the investigation of high- $T_{c}$ superconductivity (see Ref. [30] and references within). The CS Lagrangian is constructed as a gauge theory, alternative to the Maxwell Lagrangian, when one imposes Lorentz and gauge invariance besides locality, in odd dimensions:

$$
\mathcal{L}_{\mathrm{CS}}=\frac{\kappa}{2} \varepsilon^{\mu \nu \rho} A_{\mu} \partial_{\nu} A_{\rho}-A_{\mu} J^{\mu} .
$$

Note that under a gauge transformation the CS Lagrangian remains unchanged, apart from a total space-time derivative. Therefore, the corresponding action $S_{\mathrm{CS}}=\int d^{3} x \mathcal{L}_{\mathrm{CS}}$ is gauge invariant as long as we are allowed to neglect boundary terms.
We stress that the CS action in $2+1$ dimensions (excluding the interaction term) corresponds to the dimensionally reduced topological $\theta$ term composed of the field strength and its dual in four dimensions:

$$
S_{\theta}^{4 D}=\frac{\theta}{4} \int d^{4} x F_{\mu \nu} \tilde{F}_{\mu \nu} .
$$

As described in Ref. [39], it corresponds to the integral of a total derivative:

$$
S_{\theta}^{4 D}=\frac{\theta}{4} \int d^{4} x \partial_{\mu} I^{\mu}, \quad I^{\mu}=\epsilon^{\mu \nu \alpha, \beta} A_{\nu} \partial_{\alpha} A_{\beta} .
$$

Although the contribution of such a term is trivial for Abelian theories in four dimensions, this is not the case in $2+1$ D. Applying Gauss' theorem to Eq. (9) yields the action

$$
S_{\theta}^{3} D=-\frac{\theta}{2}\left[\int_{z_{3}=0} d^{2} \xi^{\mu} I_{\mu}+\int_{S_{\infty}} d^{3} \xi^{\mu} I_{\mu}\right] .
$$

Noting that for the first term $d^{3} \xi=-d x^{0} d x^{1} d x^{2}$ and taking the convention $\epsilon^{0123}=\epsilon_{3012}=1$ and $\epsilon^{012}=\epsilon_{012}=1$, the reduced version of the action in Eq. (8) is

$$
S_{\theta}^{3 D}=\frac{\theta}{2} \int d^{3} x \epsilon^{\mu \alpha \beta} A_{\mu} \partial_{\alpha} A_{\beta}
$$

if boundary terms can be neglected. This corresponds to the CS term considered here.

In order to explore the influence of a CS term on the chiral symmetry, we add it to the Lagrangian (4), yielding

$$
\begin{aligned}
\mathcal{L}_{\mathrm{RQED}}^{\mathrm{CS}}= & -\frac{1}{4} F^{\mu \nu} \frac{2}{(-\square)^{1 / 2}} F_{\mu \nu}+\bar{\psi}\left(i \gamma^{\mu} \partial_{\mu}+e \gamma^{\mu} A_{\mu}\right) \psi \\
& +\frac{1}{2 \zeta}(\partial \cdot A)^{2}+\frac{-i \theta}{4} \varepsilon^{\mu \nu \rho} A_{\mu} \partial_{\nu} A_{\rho} .
\end{aligned}
$$

The bare photon propagator derived from the above Lagrangian can be written as [51]

$$
\begin{aligned}
\hat{\Delta}_{\mu \nu}(\vec{q})= & \frac{1}{2 q} \frac{1}{\left(1+\theta^{2}\right)}\left(\delta_{\mu \nu}-\frac{q_{\mu} q_{\nu}}{q^{2}}\right) \\
& +\frac{\zeta}{q^{2}} \frac{q_{\mu} q_{\nu}}{q^{2}}-\frac{1}{2 q^{2}} \frac{\theta}{\left(1+\theta^{2}\right)} \epsilon_{\mu \nu \rho} q^{\rho} .
\end{aligned}
$$

We can verify from the above expression that the $\theta$ parameter is dimensionless so, in contrast to $\mathrm{QED}_{3}$, it cannot be associated to the so-called topological photon mass. ${ }^{1}$ This can be inferred from the fact that $\mathrm{P}(\mathrm{R}) \mathrm{QED}$

\footnotetext{
${ }^{1}$ Actually, in the Abelian case there is no real topology involved and the topological term is used for historical reasons based on its non-Abelian counterpart.
} 
possesses the remarkable property of scale invariance [52]. Compared to the propagator of pure $\mathrm{P}(\mathrm{R}) \mathrm{QED}$ [Eq. (5)], the presence of a CS term induces a $T$-odd contribution in the propagator, besides a normalization of the propagator. This is explicitly reflected in the mass generation we present below in Sec. III.

\section{NONPERTURBATIVE SOLUTION}

In order to explore the dynamical generation of fermion mass in $\mathrm{R}(\mathrm{P}) \mathrm{QED}$, we work within the SDE framework, which has been used to address the issue of CSB in QCD (see Refs. [53-56] for recent reviews). Such a framework consists of an infinite tower of coupled equations among the $n$-point Green functions of the theory. The corresponding equations for the two-point functions are

$$
\begin{gathered}
S^{-1}(p)=S_{0}^{-1}(p)-\Xi(p), \\
\Delta_{\mu \nu}^{-1}(p)=\Delta_{0 \mu \nu}^{-1}(p)-\Pi_{\mu \nu}(p),
\end{gathered}
$$

corresponding to the fermion and photon propagators, respectively. The index 0 stands for the bare propagator and $\Xi(p)$ and $\Pi_{\mu \nu}(p)$ are the fermion and photon selfenergies. In Ref. [48], a truncation of the system of SD equations is considered including vacuum polarization effects and the central Ball-Chiu vertex, including the full set of dressing functions. Due to the lack of a pointwise evolution for the solutions of the SDEs with the coupling and CS parameter, in what follows we work in the quenched limit, neglecting fermion-loop contributions for the vacuum polarization. Besides that, we use the rainbowladder truncation - the bare fermion-photon vertex-to decouple the SD equations. This is a common truncation adopted in the literature to capture the traits of CSB in different quantum field theories. A more refined truncation scheme demands satisfaction of the underlying symmetries of $\mathrm{R}(\mathrm{P}) \mathrm{QED}$. The general solution to the SDE for the fermion propagator in Eq. (15) is

$$
S^{-1}(p)=-A(p) \gamma^{\mu} p_{\mu}+\Sigma(p) .
$$

We adopt the rainbow-ladder truncation by selecting the tree-level fermion-photon vertex and photon propagator. Furthermore, we neglect the wave function renormalization, setting $A(p)=1$. In this fashion, we have the following form for the mass function:

$$
\Sigma(p)=4 \pi \alpha \int \frac{d^{3} k}{(2 \pi)^{3}} \frac{\Sigma(k)}{k^{2}+\Sigma^{2}(k)} \frac{1}{q},
$$

where $\alpha=e^{2} / 4 \pi$ is the coupling. Notice that this equation has the same functional form as Eq. (22) of Ref. [40]. However, that equation was derived on very different grounds. It was obtained by including vacuum polarization effects at the leading order of the $1 / N_{f}$ approximation and working in a nonlocal gauge. As such, the coupling

$$
\alpha \rightarrow \lambda=\frac{4 \alpha}{3\left(1+\frac{\pi \alpha N_{f}}{4}\right)}
$$

can now serve to identify the critical number of fermion families $N_{f}$ for chiral symmetry restoration.

When including the CS term, it is interesting to work with projected right- and left-handed fermions as $\psi_{ \pm}=\chi_{ \pm} \psi$. Given $\tau=\left[\gamma_{3}, \gamma_{5}\right] / 2$, the chiral projector is defined as $\chi_{ \pm}=(1 \pm \tau) / 2$, which implies the following properties: $\chi_{ \pm}^{2}=\chi_{ \pm}, \chi_{+} \chi_{-}=0$, and $\chi_{+}+\chi_{-}=1$. The advantage of this procedure is the following: in order to explore the nonperturbative mass generation we allow for the general case where an ordinary Dirac mass $m_{e} \bar{\psi} \psi$ can emerge. On top of that, there is another mass term that potentially may be generated and is particularly interesting to us, known as the Haldane mass $m_{o} \bar{\psi} \tau \psi$, with $\tau=\left[\gamma_{3}, \gamma_{5}\right] / 2$. This term does not break chiral symmetry, but rather violates parity (and time reversal), and because the CS is parity violating we expect a relation between them. On the other hand, the Dirac mass behaves in the opposite way, preserving $P$ and $T$ symmetry but, of course, breaking chiral symmetry. With these considerations, the most general Lagrangian we consider for our analysis is

$$
\begin{aligned}
\mathcal{L}_{\mathrm{RQED}}^{\mathrm{CS}}= & -\frac{1}{4} F^{\mu \nu} \frac{2}{(-\square)^{1 / 2}} F_{\mu \nu}+\bar{\psi}\left(i \gamma^{\mu} \partial_{\mu}+e \gamma^{\mu} A_{\mu}\right. \\
& \left.+m_{e}+\tau m_{o}\right) \psi+\frac{1}{2 \zeta}(\partial \cdot A)^{2}+\frac{-i \theta}{4} \varepsilon^{\mu \nu \rho} A_{\mu} \partial_{\nu} A_{\rho},
\end{aligned}
$$

from which the fermion propagator has the structure

$S_{F}^{-1}(p)=A_{e}(p) \not p+A_{o}(p) \tau \not p-B_{e}(p)-B_{o}(p) \tau$.

However, these masses do not correspond to poles in the propagator. The operators $\chi_{ \pm}$project the upper and lower two-component spinors, making explicit the poles of each fermion species. Acting with the chiral projectors on the fermion sector, we have

$$
\mathcal{L}_{F}=\bar{\psi}_{+}\left(i \not \partial-M_{+}\right) \psi_{+}+\bar{\psi}_{-}\left(i \not \partial-M_{-}\right) \psi_{-},
$$

where $M_{+}=m_{e}+m_{o}$ and $M_{-}=m_{e}-m_{o}$.

Given that, we start from the gap equation

$S_{ \pm}^{-1}(p)=S_{0 \pm}^{-1}(p)+4 \pi \alpha \int \frac{d^{3} k}{(2 \pi)^{3}} \gamma^{\mu} S_{ \pm}(k) \gamma^{\nu} \Delta_{\mu \nu}(q)$,

where

$$
\begin{aligned}
S_{ \pm}^{-1}(p) & =\left(\not p+M_{ \pm}(p)\right) \chi_{ \pm}, \\
S_{0}^{-1}(p) & =\not \not \chi_{ \pm}, \\
S_{ \pm}(k) & =-\frac{\not k+M_{ \pm}(k)}{k^{2}+M_{ \pm}^{2}(k)} \chi_{ \pm},
\end{aligned}
$$


and $\Delta_{\mu \nu}$ is the photon propagator given by Eq. (13). Inserting these equations into Eq. (21) and taking the traces, we reach the following gap equation:

$$
\begin{aligned}
M_{ \pm}(p)= & 2 \pi \alpha \int \frac{d^{3} k}{(2 \pi)^{3}}\left[\frac{2 M_{ \pm}(k)}{k^{2}+M_{ \pm}^{2}(k)} \frac{1}{q\left(1+\theta^{2}\right)}\right. \\
& \left.\mp \frac{1}{q^{2}} \frac{\theta}{1+\theta^{2}} \frac{k \cdot q}{k^{2}+M_{ \pm}^{2}(k)}\right] .
\end{aligned}
$$

Performing a straightforward and standard calculation (detailed in the Appendix B), the gap equation evolves to the differential equation

$$
\begin{gathered}
p^{2} M_{ \pm}^{\prime \prime}(p)+2 p M_{ \pm}^{\prime}(p)+\frac{2 \alpha}{\pi\left(1+\theta^{2}\right)} M_{ \pm}(p) \\
=\mp \frac{2 \alpha \theta}{\pi\left(1+\theta^{2}\right)}\left[\frac{5 p}{3}-\frac{6 p^{2}}{\kappa}+\frac{\Lambda^{3}}{9 p^{2}}-\frac{p}{9}\right]
\end{gathered}
$$

with the boundary conditions

$$
\left.p^{2} M^{\prime}(p)\right|_{p \rightarrow 0}=0,\left.\quad\left(p M^{\prime}(p)+M(p)\right)\right|_{p=\Lambda}=0,
$$

whose solution is given by

$$
\begin{aligned}
M_{ \pm}(p)= & p^{-\frac{1}{2} \sqrt{1-\frac{\alpha}{\alpha_{c}}}-\frac{1}{2}}\left(c_{2} p^{\sqrt{1-\frac{\alpha}{\alpha_{c}}}}+c_{1}\right) \\
& +f(\theta, p) \\
= & \Lambda e^{-\pi / \sqrt{\alpha / \alpha_{c}-1}}+f(\theta, p),
\end{aligned}
$$

where

$$
\alpha_{c}=\frac{\pi}{8}\left(1+\theta^{2}\right)
$$

and

$$
\begin{aligned}
f(\theta, p)= & {\left[\pi ( \theta ^ { 2 } + 1 ) ( \mp \frac { 2 \alpha \theta } { \theta ^ { 2 } + 1 } ) \left(\kappa\left(\alpha+3 \pi\left(\theta^{2}+1\right)\right)\right.\right.} \\
& \left.\left.\times\left(\Lambda^{3}+14 p^{3}\right)-54 p^{4}\left(\alpha+\pi\left(\theta^{2}+1\right)\right)\right)\right] \\
& \times \frac{1}{18 \kappa p^{2}\left(\alpha+\pi\left(\theta^{2}+1\right)\right)\left(\alpha+3 \pi\left(\theta^{2}+1\right)\right)} .
\end{aligned}
$$

We can identify the Miransky scaling law in the first term of the second row of Eq. (26). This term corresponds to the solution of ordinary R(P)QED obtained from a homogeneous version of the differential equation (24). The only difference from the first piece in the present solution to the solution of pure $\mathrm{R}(\mathrm{P}) \mathrm{QED}$ is a rescaling of the critical coupling as in Eq. (27). It can be verified that in the limit $\theta \rightarrow 0$, we recover the usual critical coupling. Thus, we observe the role of the CS coefficient $\theta$ as an effective dielectric constant [47]. Furthermore, the inhomogeneity on the right-hand side of Eq. (24), brought by the extra CS piece in the photon propagator (13), is responsible for the emergence of $f(\theta, p)$.

In Figs. 1 and 2 we plot $M_{-}$and $M_{+}$as functions of the external momentum $p$, respectively, for several values of $\theta=\eta \theta_{c}$, with $0 \leq \eta \leq 1$ for fixed $\alpha=1.07 \alpha_{c}$, which corresponds to $\theta_{c}=6.6 \times 10^{-14}$. We notice that for small momentum both masses are finite and approximately $p$ independent. For larger momentum, the height of the mass functions start to decrease. This remains true for different values of $\theta$ as long as the coupling considered is above its critical value. For couplings below $\alpha_{c}$, the solution oscillates very rapidly around zero, indicating that the masses are vanishing. Therefore, Figs. 1 and 2 indicate that masses can be dynamically generated in CS-R(P)QED, and their magnitude is $\theta$ dependent. This dependence is opposite for $M_{+}$and $M_{-}$: while the value of $M_{-}$increases as $\theta$ increases, $M_{+}$decreases, reaching a critical point at $\theta_{c}$ when it abruptly changes sign, signaling a first-order phase transition. In order to examine the consequences on the chiral and parity symmetries we must check how this behavior is reflected in $m_{e}$ and $m_{o}$.

The heights of the mass functions in the deep infrared, $M_{ \pm}(0)$, which measure the amount of dynamically generated mass, are depicted in Figs. 3 and 4 for a fixed value of $\alpha=x \alpha_{c}$. Above $\theta_{c}$, the value of $M_{+}(0)$ undergoes a discontinuity and jumps, assuming negative values. In Fig. 3 we have flipped the sign for $M_{+}$above the discontinuity to lead the eye. In these figures we can clearly see the opposite behavior of the poles of right- and left-handed fermions as $\theta$ increases and appreciate the discontinuity of $M_{+}$as the smoking gun of a first-order phase transition. For a better perspective and comparison between $M_{+}$and $M_{-}$, we have rescaled them by their values at $\theta=0$ and combined their plots in Fig. 5. As $\theta$ increases the masses split, and at a critical $\theta, M_{+}$undergoes a discontinuity and changes sign. The red dots show the absolute values of $M_{+}$above $\theta_{c}$. For this range, the two

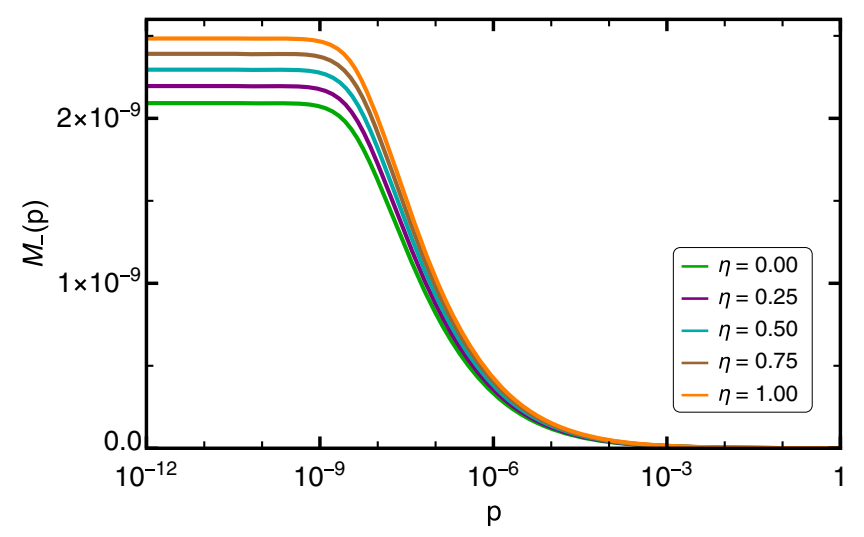

FIG. 1. Mass function $M_{-}$plotted as a function of momentum. The different curves correspond to different values of the CS parameter $\theta=\eta \theta_{c}$, with $0 \leq \eta \leq 1$ and a fixed value of the coupling $\alpha=1.07 \alpha_{c}$. 


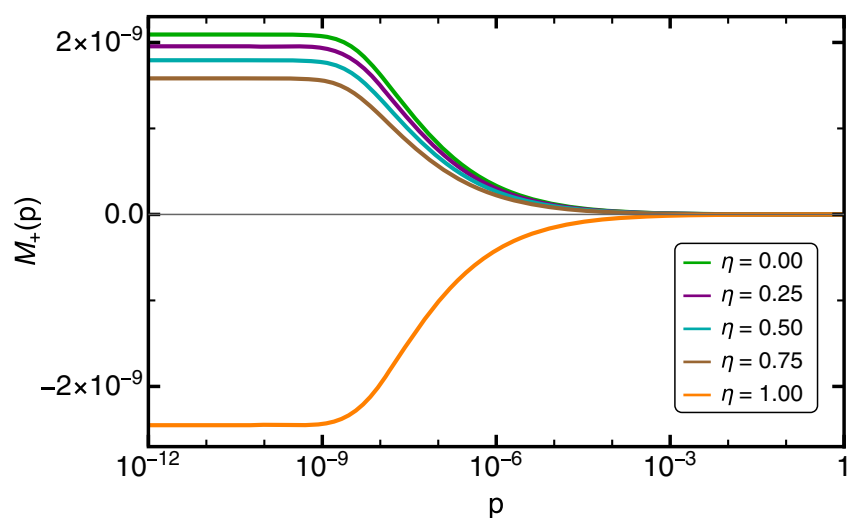

FIG. 2. Mass function $M_{+}$plotted as a function of momentum. The different curves correspond to different values of the CS parameter $\theta=\eta \theta_{c}$, with $0 \leq \eta \leq 1$ and fixed value of the coupling $\alpha=1.07 \alpha_{c}$.

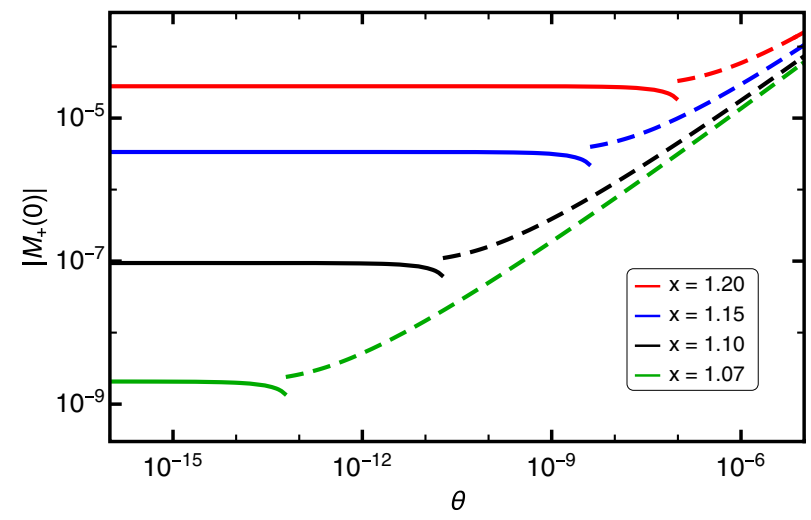

FIG. 3. The absolute value of the pole of the right fermion propagator $M_{+}$plotted as a function of the CS parameter $\theta$. Above $\theta_{c}$ the mass $M_{+}$becomes negative, and here we flipped its sign for better visualization. The different curves correspond to different values of the coupling $\alpha=x \alpha_{c}$.

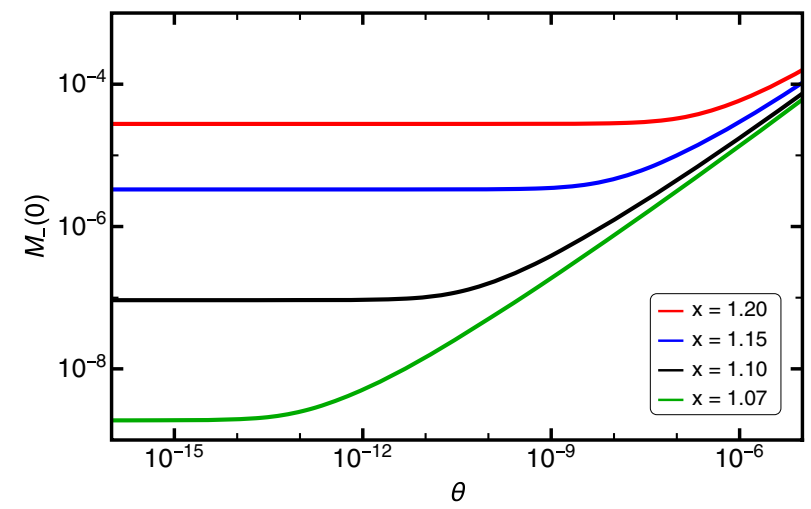

FIG. 4. The pole of the left fermion propagator $M_{-}$plotted as a function of the CS parameter $\theta$. The different curves correspond to different values of the coupling $\alpha=x \alpha_{c}$.

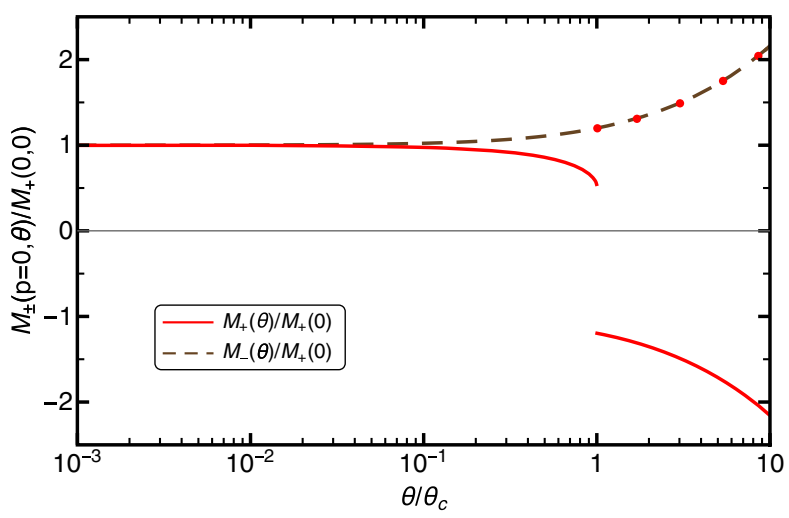

FIG. 5. $M_{ \pm}$rescaled by their $\theta=0$ values. The full line corresponds to $M_{+}$and the dashed line to $M_{-}$. The red dots are the absolute values of $M_{+}$above the critical $\theta$.

masses become mirror images of one another. We notice that since the curves are rescaled, they coincide for any value of $x>1$.

The parity-even and parity-odd masses that appear in the Lagrangian (19) can be obtained by summing or subtracting $M_{ \pm}$, respectively. We can verify in Figs. 6 and 7 that both $m_{e}$ and $m_{o}$ are nonvanishing for a large range of $\theta$, clearly showing that CSB can occur within this model. For small $\theta$ the Dirac mass $m_{e}$ (responsible for breaking chiral symmetry) exhibits a plateau, leaping at a critical $\theta$ to a value several orders of magnitude smaller. After that, $m_{e}$ increases again and finally drops to zero. We interpret the discontinuity as an attempt to restore chiral symmetry, associated to the first piece in Eq. (23). That would be the critical point if $f(\theta, 0)$ was absent. However, as one moves to the right of the discontinuity, the CS contribution $f(\theta, 0)$ becomes dominant and $m_{e}$ starts to be significant again. For even larger $\theta$ the Dirac mass drops and the real critical $\theta$, for which chiral symmetry is restored, is around $\theta=1$. In turn, parity breaking is encoded in $m_{o}$. As the CS parameter becomes dominant, the Haldane mass increases, never restoring the symmetry.

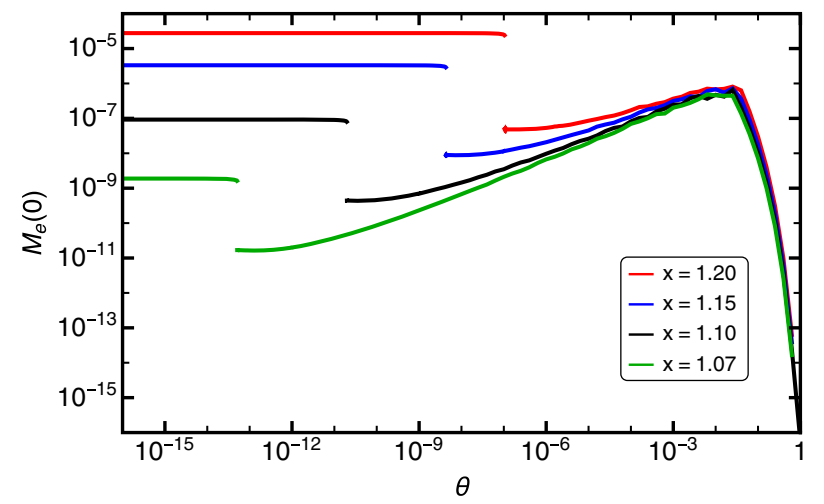

FIG. 6. Dynamical mass term, even under parity transformation, as a function of the CS parameter $\theta$. 


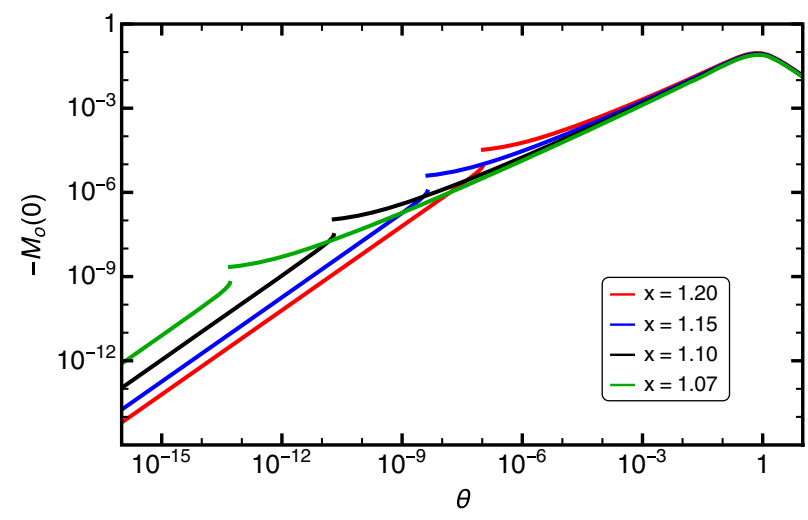

FIG. 7. Dynamical mass term, odd under parity transformation, as a function of the CS parameter $\theta$.

\section{FINAL REMARKS}

In this paper we have investigated the chiral symmetry breaking and restoration in the framework of pseudo QED (a.k.a. reduced QED) with a CS term. This is a mixeddimensional theory applicable to systems where fermions are constrained to a plane while gauge fields are free to live in the bulk. The interaction of charge carriers in planar Dirac/Weyl materials and external electromagnetic fields in general must be described by this type of theories.

We have calculated the radiative correction due to the CS term, and suitably nonperturbatively truncated the SDE in order to explore the dynamical mass generation in the model. We worked in the quenched approximation and used the rainbow-ladder truncation. In order to explore the CSB we searched for two types of masses: the Dirac mass $m_{e} \bar{\psi} \psi$ and the Haldane mass $m_{o} \bar{\psi} \tau \psi$. These bilinears are such that while the Dirac mass breaks chiral symmetry and preserves parity and time reversal, the Haldane mass leaves the chiral symmetry intact and violates these discrete symmetries.

We have shown that both masses appear for small values of $\theta$, provided the coupling considered is above the critical coupling $\alpha=\pi / 8$. A competition between the pure $\mathrm{P}(\mathrm{R})$ QED and the effect of the CS term takes place, with the latter giving contributions for both masses. For large enough $\theta$, the chiral symmetry is finally restored, while the Haldane mass remains finite and the breaking of $P$ symmetry persists for the entire range of $\theta$ considered here.

This result is especially relevant for what concerns chiral currents in planar materials. The chiral magnetic effect, initially proposed to take place in the quark-gluon plasma formed in heavy-ion collisions [57,58], was observed in three-dimensional Dirac materials [59]. This was possible since the charge carriers in these kinds of materials have a relativistic-like behavior, allowing for a connection with high-energy physics. The gapless nature of these quasiparticles makes their dispersion relation identical to that of a massless free fermion. When an imbalance of chirality takes place, an anomalous electric current is generated in the direction of an applied external magnetic field. In the quark-gluon plasma it is argued that the quantum anomaly responsible for this imbalance is originated from topological configurations of the gauge fields. In $(3+1) \mathrm{D}$ materials, the chiral anomaly is neatly generated by simultaneously applying aligned electric and magnetic fields.

It was considered by some of us that the chiral magnetic effect could happen in another class of materials, with potential advantages [60,61]: planar materials organized into honeycomb lattices. The standard way to model this type of structure is to work with two triangular sublattices and expand the energy around zero-energy points, known as Dirac points. The two sublattices and two nontrivial Dirac points make a four-component spinor and produce an analog to the chirality. It was shown in Refs. $[60,61]$ that as long as the fermion sector contains Dirac and Haldane masses, the chiral magnetic effect might occur in these semimetals.

The dynamically generated masses obtained in the present work correspond to the ones considered in Refs. [60,61]. The CS term has been shown to be capable of giving rise to Dirac and Haldane masses. Considerations excluding the possibility of the chiral magnetic effect in planar materials were taken within QED strictly in three space-time dimensions, while a suitable theory to model real materials immersed in external fields must be mixed dimensional. Since parallel electric and magnetic fields correspond to a CS term, it might be possible that in-plane collinear fields applied in honeycomb lattice samples, like graphene, could generate the desired gap to work out the chiral magnetic effect.

We believe that the contribution of our results is twofold: as a toy model to investigate anomaly effects in the chiral symmetry breaking of QCD, and as a model to describe gap generation in planar Dirac materials that happen via interactions with external electromagnetic fields. The robustness of our findings against a more refined truncation of the SD equations along the lines of that proposed in Ref. [48] is currently under scrutiny. Results shall be presented elsewhere.

\section{ACKNOWLEDGMENTS}

A. J. M. receives partial support from FAPESP under fellowship number 2016/12705-7, and L. A. is supported under the fellowship number 2018/17643-5.

\section{APPENDIX A: SDE}

Although the effective action of $\mathrm{R}(\mathrm{P}) \mathrm{QED}$ is highly nonlocal, the corresponding propagators are well behaved. Accordingly, the gap equation in R(P)QED can be straightforwardly derived from the standard Feynman rules of perturbation theory derived directly from the Lagrangian and the multiloop Gegenbauer polynomial technique. Alternatively, one can merely exploit the correspondence between this theory and ordinary QED in three dimensions, $\mathrm{QED}_{3}$, with a large number of fermion families [62]. 


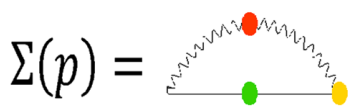

FIG. 8. Fermion self-energy.

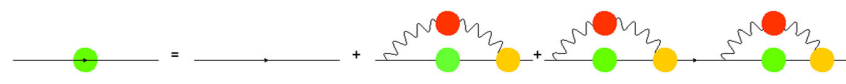

FIG. 9. Perturbative expansion of the fermion propagator in terms of the self-energy.

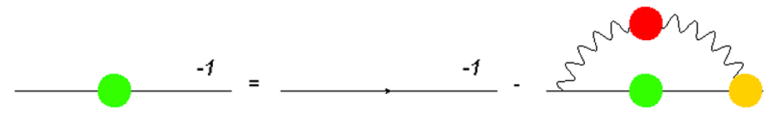

FIG. 10. SDE for the inverse Fermion propagator.

From the perturbative perspective, the complete fermion two-point Green function can be constructed from the selfenergy, which includes all radiative corrections and is depicted in Fig. 8.

In terms of $\Sigma(p)$, the perturbative expansion of the fermion propagator is shown in Fig. 9.

It corresponds to the expansion

$$
\begin{aligned}
S(p)= & S_{0}(p)+S_{0}(p) \Sigma(p) S_{0}(p) \\
& +S_{0}(p) \Sigma(p) S_{0}(p) \Sigma(p) S_{0}(p)+\ldots \\
= & S_{0}(p)+S_{0}(p) \Sigma(p)\left[S_{0}(p)+S_{0}(p) \Sigma(p) S_{0}(p)+\ldots\right] \\
= & S_{0}(p)+S_{0}(p) \Sigma(p) S(p) .
\end{aligned}
$$

This is the SDE for the fermion propagator. It is convenient to write the equation corresponding to the inverse of this propagator, which explicitly reads

$$
S^{-1}(p)=\left(S_{0}(p)\right)^{-1}-\Sigma(p) .
$$

This is the SDE for the two-point Green function and is represented in Fig. 10.

\section{APPENDIX B: TRACE THEOREMS AND ANGULAR INTEGRALS FOR THE GAP EQUATION}

For the chiral projectors we have the following trace theorems:

$$
\begin{aligned}
\operatorname{Tr}\left[\chi_{ \pm}\right] & =2, \\
\operatorname{Tr}\left[\gamma_{\mu} \chi_{ \pm}\right] & =0, \\
\operatorname{Tr}\left[\gamma_{\mu} \gamma_{\nu} \chi_{ \pm}\right] & =-2 \delta_{\mu \nu}, \\
\operatorname{Tr}\left[\gamma_{\mu} \gamma_{\nu} \gamma_{\rho} \chi_{ \pm}\right] & =\mp 2 \epsilon_{\mu \nu \rho}, \\
\operatorname{Tr}\left[\gamma_{\mu} \gamma_{\nu} \gamma_{\rho} \gamma_{\sigma} \chi_{ \pm}\right] & =2\left(\delta_{\mu \nu} \delta_{\rho \sigma}-\delta_{\mu \rho} \delta_{\nu \sigma}+\delta_{\mu \sigma} \delta_{\nu \rho}\right) .
\end{aligned}
$$

The angular integral

$$
I_{1}=2 \int_{0}^{\pi} \frac{d \theta \sin \theta}{\sqrt{k^{2}+p^{2}-2 k p \cos \theta}}
$$

is written as

$$
\begin{aligned}
I_{1}= & \int_{-1}^{1} \frac{d x}{\sqrt{k^{2}+p^{2}-2 k p x}} \\
= & \frac{\theta(k-p)}{k} \int_{-1}^{1} \frac{d x}{\sqrt{1+\frac{p^{2}}{k^{2}}-2 \frac{p}{k} x}} \\
& +\frac{\theta(p-k)}{p} \int_{-1}^{1} \frac{d x}{\sqrt{1+\frac{k^{2}}{p^{2}}-2 \frac{k}{p} x}} .
\end{aligned}
$$

Then, it is simplified by the identity

$$
\int_{-1}^{1} \frac{d x}{\sqrt{1-a^{2}-2 a x}}=2
$$

valid for $0<a<1$. The angular integral

$$
\begin{aligned}
I_{2} & =\int_{0}^{\pi} \frac{d \theta \sin \theta k \cdot q}{k^{2}+p^{2}-2 k p \cos \theta} \\
& =\int_{0}^{\pi} \frac{d \theta \sin \theta\left(k^{2}-k p \cos \theta\right)}{k^{2}+p^{2}-2 k p \cos \theta}
\end{aligned}
$$

can be conveniently written as

$$
\begin{aligned}
I_{2}= & k^{2}\left[\frac{\theta(k-p)}{k^{2}} \int_{-1}^{1} \frac{d x}{1+\frac{p^{2}}{k^{2}}-2 \frac{p}{k} x}\right. \\
& \left.+\frac{\theta(p-k)}{p^{2}} \int_{-1}^{1} \frac{d x}{1+\frac{k^{2}}{p^{2}}-2 \frac{k}{p} x}\right] \\
& -k p\left[\frac{\theta(k-p)}{k^{2}} \int_{-1}^{1} \frac{d x x}{1+\frac{p^{2}}{k^{2}}-2 \frac{p}{k} x}\right. \\
& \left.+\frac{\theta(p-k)}{p^{2}} \int_{-1}^{1} \frac{d x x}{1+\frac{k^{2}}{p^{2}}-2 \frac{k}{p} x}\right] .
\end{aligned}
$$

Then, the final form of the gap equation is obtained from

$$
\int_{-1}^{1} \frac{d x}{1+a^{2}-2 a x} \approx 2
$$

and

$$
\int_{-1}^{1} \frac{d x x}{1+a^{2}-2 a x} \approx \frac{4 a}{3},
$$

where the asymptotic behavior is reached as $(a \rightarrow 0)$. 
[1] M. Göpfert and G. Mack, Commun. Math. Phys. 82, 545 (1982).

[2] C. J. Burden, J. Praschifka, and C. D. Roberts, Phys. Rev. D 46, 2695 (1992).

[3] T. W. Appelquist, M. J. Bowick, D. Karabali, and L. Wijewardhana, Phys. Rev. D 33, 3704 (1986).

[4] R. D. Pisarski, Phys. Rev. D 29, 2423 (1984).

[5] T. Appelquist, D. Nash, and L. Wijewardhana, Phys. Rev. Lett. 60, 2575 (1988).

[6] D. Nash, Phys. Rev. Lett. 62, 3024 (1989).

[7] D. Atkinson, P. Johnson, and P. Maris, Phys. Rev. D 42, 602 (1990).

[8] C. J. Burden and C. D. Roberts, Phys. Rev. D 44, 540 (1991).

[9] M. Pennington and D. Walsh, Phys. Lett. B 253, 246 (1991).

[10] C. J. Burden, J. Praschifka, and C. D. Roberts, Phys. Rev. D 46, 2695 (1992).

[11] V. Gusynin, A. Hams, and M. Reenders, Phys. Rev. D 53, 2227 (1996).

[12] V. Gusynin, A. Hams, and M. Reenders, Phys. Rev. D 63, 045025 (2001).

[13] A. Bashir and A. Raya, Phys. Rev. D 64, 105001 (2001).

[14] A. Bashir, A. Huet, and A. Raya, Phys. Rev. D 66, 025029 (2002).

[15] A. Bashir and A. Raya, Phys. Rev. D 66, 105005 (2002).

[16] V. Gusynin and M. Reenders, Phys. Rev. D 68, 025017 (2003).

[17] C. Fischer, R. Alkofer, T. Dahm, and P. Maris, Phys. Rev. D 70, 073007 (2004).

[18] A. Bashir and A. Raya, Nucl. Phys. B709, 307 (2005).

[19] A. Bashir and A. Raya, Few Body Syst. 41, 185 (2007).

[20] A. Bashir, A. Raya, I. Cloet, and C. Roberts, Phys. Rev. C 78, 055201 (2008).

[21] A. Bashir, A. Raya, S. Sanchez-Madrigal, and C. Roberts, Few Body Syst. 46, 229 (2009).

[22] A. Bashir, A. Raya, and S. Sanchez-Madrigal, Phys. Rev. D 84, 036013 (2011).

[23] K. Raya, A. Bashir, S. Hernndez-Ortiz, A. Raya, and C. Roberts, Phys. Rev. D 88, 096003 (2013).

[24] V. Gusynin and P. Pyatkovskiy, Phys. Rev. D 94, 125009 (2016).

[25] A. Kotikov, V. Shilin, and S. Teber, Phys. Rev. D 94, 056009 (2016).

[26] A. V. Kotikov and S. Teber, Particles 3, 345 (2020).

[27] S. J. Hands, J. B. Kogut, L. Scorzato, and C. G. Strouthos, Phys. Rev. B 70, 104501 (2004).

[28] A. Khare, Fractional Statistics and Quantum Physics, 2nd ed. (World Scientific, London, 2005).

[29] A. Lerda, Anyons. Quantum Mechanics of Particles with Fractionary Statistics, Lecture Notes in Physics (SpringerVerlag, Berlin, Heidelberg, New York, 1992).

[30] Fractional Statistics and Anyon Superconductivity, edited by F. Wilczek, Series on Directions on Condensed Matter Physics (World Scientific, Singapore, 1990).

[31] K. I. Kondo and P. Maris, Phys. Rev. Lett. 74, 18 (1995).

[32] K. I. Kondo and P. Maris, Phys. Rev. D 52, 1212 (1995).

[33] P. Maris, Phys. Rev. D 54, 4049 (1996).
[34] C. P. Hofmann, A. Raya, and S. S. Madrigal, Phys. Rev. D 82, 096011 (2010).

[35] V. P. Gusynin, S. G. Sharapov, and J. P. Carbotte, Int. J. Mod. Phys. B 21, 4611 (2007).

[36] S. Cahangirov, H. Sahin, G. Le Lay, and A. Rubio, Introduction to the Physics of Silicene and Other 2D Materials, Lecture Notes in Physics (Springer Internationa Publishing AG, New York, 2017).

[37] M. I. Katsnelson, K. S. Novoselov, and A. K. Geim, Nat. Phys. 2, 620 (2006).

[38] M. Katsnelson, Eur. Phys. J. B 51, 157 (2006).

[39] E. C. Marino, Nucl. Phys. B408, 551 (1993).

[40] E. V. Gorbar, V. P. Gusynin, and V. A. Miransky, Phys. Rev. D 64, 105028 (2001).

[41] L. O. Nascimento, V. Alves, F. Pea, C. M. Smith, and E. C. Marino, Phys. Rev. D 92, 025018 (2015).

[42] V. Alves, W. S. Elias, L. O. Nascimento, V. Jurii, and F. Pea, Phys. Rev. D 87, 125002 (2013).

[43] R. Fukuda and T. Kugo, Nucl. Phys. B117, 250 (1976).

[44] V. A. Miransky, Phys. Lett. 165B, 401 (1985).

[45] P. I. Fomin, V. P. Gusynin, V. A. Miransky, and Y. A. Sitenko, Riv. Nuovo Cimento 6, 1 (1983).

[46] J. Báez, A. Raya, and J. C. Rojas, Phys. Rev. D 102, 056020 (2020).

[47] G. C. Magalhães, V. S. Alves, E. C. Marino, and L. O. Nascimento, Phys. Rev. D 101, 116005 (2020).

[48] M. E. Carrington, Phys. Rev. B 99, 115432 (2019).

[49] R. L. P. G. do Amaral and E. C. Marino, J. Phys. A 25, 5183 (1992).

[50] M. de Jesus Anguiano Galicia and A. Bashir, Few Body Syst. 37, 71 (2005).

[51] D. Dudal, A. J. Mizher, and P. Pais, Phys. Rev. D 98, 065008 (2018).

[52] D. Dudal, A. J. Mizher, and P. Pais, Phys. Rev. D 99, 045017 (2019).

[53] C. D. Roberts, Symmetry 12, 1468 (2020).

[54] A. Aguilar et al., Eur. Phys. J. A 55, 190 (2019).

[55] C. S. Fischer, Prog. Part. Nucl. Phys. 105, 1 (2019).

[56] T. Horn and C. D. Roberts, J. Phys. G 43, 073001 (2016).

[57] D. E. Kharzeev, L. D. McLerran, and H. J. Warringa, Nucl. Phys. A803, 227 (2008).

[58] K. Fukushima, D. E. Kharzeev, and H. J. Warringa, Phys. Rev. D 78, 074033 (2008).

[59] Q. Li, D. E. Kharzeev, C. Zhang, Y. Huang, I. Pletikosić, A. V. Fedorov, R. D. Zhong, J. A. Schneeloch, G. D. Gu, and T. Valla, Nat. Phys. 12, 550 (2016); G. Zheng et al., Phys. Rev. B 93, 115414 (2016); C.-Z. Li, L.-X. Wang, H. Liu, J. Wang, Z.-M. Liao, and D.-P. Yu, Nat. Commun. 6, 10137 (2015); X.Huang et al., Phys. Rev. X 5, 031023 (2015); Z. Wang et al., Phys. Rev. B 93, 121112 (2016); F. Arnold et al., Nat. Commun. 7, 11615 (2016); C. Zhang, E. Zhang, W. Wang et al., Nat. Commun. 8, 13741 (2017).

[60] A. J. Mizher, S. Hernandez-Ortiz, A. Raya, and C. Villavicencio, Eur. Phys. J. C 78, 912 (2018).

[61] A. J. Mizher, A. Raya, and C. Villavicencio, Int. J. Mod. Phys. B 30, 1550257 (2016).

[62] A. V. Kotikov and S. Teber, Phys. Rev. D 94, 114010 (2016). 